\title{
Correction to: Long- and Mid-Term Variations of the Soft X-ray Flare Character in Solar Cycles
}

\author{
I.M. Chertok ${ }^{1}$ - A.V. Belov ${ }^{1}$
}

Published online: 20 February 2018

(C) Springer Science+Business Media B.V., part of Springer Nature 2018

\section{Correction to: Solar Phys (2017) 292, 114 \\ https://doi.org/10.1007/s11207-017-1169-1}

We found an important error in the text of our article.

On page 6, the second sentence of Section 3.2

"We studied the variations in soft X-ray flare characteristics in more detail by averaging them within the running windows of \pm one Carrington rotation with a step of two rotations."

should instead read

"We studied the variations in soft X-ray flare characteristics in more detail by averaging them within the running windows of \pm 2.5 Carrington rotations with a step of two rotations."

We regret the inconvenience.

The online version of the original article can be found under https://doi.org/10.1007/s11207-017-1169-1.

I.M. Chertok

ichertok@izmiran.ru

A.V. Belov

abelov@izmiran.ru

1 Pushkov Institute of Terrestrial Magnetism, Ionosphere and Radio Wave Propagation (IZMIRAN), Troitsk, Moscow, 108840 Russia 